\title{
Cancer Nanomedicine: From Targeted Delivery to Combination Therapy
}

\section{Citation}

Xu, Xiaoyang, William Ho, Xueqing Zhang, Nicolas Bertrand, and Omid Farokhzad. 2015. "Cancer Nanomedicine: From Targeted Delivery to Combination Therapy." Trends in molecular medicine 21 (4): 223-232. doi:10.1016/j.molmed.2015.01.001. http://dx.doi.org/10.1016/ j.molmed.2015.01.001.

\section{Published Version}

doi:10.1016/j.molmed.2015.01.001

\section{Permanent link}

http://nrs.harvard.edu/urn-3:HUL.InstRepos:26860036

\section{Terms of Use}

This article was downloaded from Harvard University's DASH repository, and is made available under the terms and conditions applicable to Other Posted Material, as set forth at http:// nrs.harvard.edu/urn-3:HUL.InstRepos:dash.current.terms-of-use\#LAA

\section{Share Your Story}

The Harvard community has made this article openly available.

Please share how this access benefits you. Submit a story.

\section{Accessibility}


Published in final edited form as:

Trends Mol Med. 2015 April ; 21(4): 223-232. doi:10.1016/j.molmed.2015.01.001.

\title{
Cancer Nanomedicine: From Targeted Delivery to Combination Therapy
}

\author{
Xiaoyang Xu $\mathbf{u}^{\mathrm{a}, \mathrm{b}, \mathrm{c}}$, William Ho ${ }^{\mathrm{a}}$, Xueqing Zhang ${ }^{\mathrm{a}}$, Nicolas Bertrand ${ }^{\mathrm{a}, \mathrm{b}}$, and Omid Farokhzad ${ }^{\mathrm{a}}$ \\ aLaboratory of Nanomedicine and Biomaterials, Brigham and Women's Hospital, Harvard Medical \\ School, Boston, MA 02115, USA \\ bThe David H. Koch Institute for Integrative Cancer Research, Massachusetts Institute of \\ Technology, Cambridge, MA 02139, USA \\ cDepartment of Chemical, Biological and Pharmaceutical Engineering, New Jersey Institute of \\ Technology, Newark, NJ 07102, USA
}

\begin{abstract}
The advent of nanomedicine marks an unparalleled opportunity to advance the treatment of a variety of diseases, including cancer. The unique properties of nanoparticles, such as large surfaceto volume ratio, small size, the ability to encapsulate a variety of drugs, and tunable surface chemistry, gives them many advantages over their bulk counterparts. This includes multivalent surface modification with targeting ligands, efficient navigation of the complex in vivo environment, increased intracellular trafficking, and sustained release of drug payload. These advantages make nanoparticles a mode of treatment potentially superior to conventional cancer therapies. This article highlights the most recent developments in cancer treatment using nanoparticles as drug-delivery vehicles, including promising opportunities in targeted and combination therapy.
\end{abstract}

\section{Keywords}

Nanomedicine; Targeted Delivery; Combination Therapy; Cancer

\section{Nanomedicine in cancer therapy}

Nanomedicine (see Glossary) is the design and development of therapeutics and diagnostic tools, distinguished by the nanoscopic scale of its delivery vehicles and diagnostic agents [1]. The nanomedical field is rapidly gaining recognition through developing ways of

(C) 2015 Published by Elsevier Ltd.

This manuscript version is made available under the CC BY-NC-ND 4.0 license.

Corresponding authors: Xu, X. (xiaoyang@njit.edu) and Farokhzad, O. (ofarokhzad@zeus.bwh.harvard.edu).

Publisher's Disclaimer: This is a PDF file of an unedited manuscript that has been accepted for publication. As a service to our customers we are providing this early version of the manuscript. The manuscript will undergo copyediting, typesetting, and review of the resulting proof before it is published in its final citable form. Please note that during the production process errors may be discovered which could affect the content, and all legal disclaimers that apply to the journal pertain.

The rest of the authors declare no conflicts of interest. 
administering treatment, particularly anticancer therapy, with unprecedented safety and efficiency. Researchers have improved upon the current standards in drug delivery relating to biodistribution, intracellular uptake, and dosing efficacy by utilizing nanoparticles (NPs) to encapsulate therapeutics and target sites of disease [2]. The successful application of processes to improve the delivery of biomedical entities through functional NPs is a revolutionary approach to disease treatment. Several liposome and polymer-based therapeutic NPs have been approved by the Food and Drug Administration (FDA) for clinical use [1]. This review will discuss the NPs under investigation with an emphasis on systems that have reached clinical trials (Table 1).

NPs are minute particles, typically less than $200 \mathrm{~nm}$ in diameter. Their nanoscopic size facilitates intracellular uptake. NPs have the ability to encapsulate therapeutics and release them in a controlled manner to specifically target diseased cells. NP encapsulation also improves the solubility of unmodified drug compounds [3]. Additional advantages of NPs have brought widespread attention to the field of nanomedicine, including their large ratio of volume-to surface area, modifiable external shell, biodegradability, and low cytotoxicity [4]. Furthermore, nanomedicine brings us dramatically closer to realizing the full promise of personalized medicine [5].

Engineered therapeutic NPs offer numerous clinical advantages. Surface modification with polyethylene glycol (PEG) protects NPs from clearance from the blood by the mononuclear phagocytic system (MPS), markedly increasing both circulation times and drug uptake by target cells $[2,6]$. Functionalization of the NP surface with multivalent targeting moieties not only improves drug efficacy but simultaneously reduces dosage, providing a novel method to optimize drug pharmacokinetics [6]. NPs spatially localize through passive/active targeting and are capable of delivering drugs through epi/endothelial barriers [3]. Below we present some examples of engineered NPs and their features that have been designed to address existing challenges in drug delivery, with a specific focus on cancer therapy.

Nanoparticles increase drug solubility, mitigate cytotoxicity, and improve drug pharmacokinetic profiles, as exemplified by nanomedicines such as Doxil ${ }^{\circledR}$ and Genexol$\mathrm{PM} \circledast$. The last decade has witnessed a number of new biotechnological approaches to the treatment of cancer. For example, the 2006 Nobel Prize in Physiology or Medicine brought renewed focus on gene silencing, and the therapeutic opportunities offered by precise regulation of gene expression have fostered the interest of medical stakeholders in siRNA and miRNA technologies [7]. Nevertheless, delivering nucleic acids into cells is challenging to say the least: nucleic acids are vulnerable to nucleases ubiquitous in the blood, and their dense negative charges hinder cell internalization. Furthermore, the non-specific interferon response triggered by the presence of foreign nucleic acids in the cytoplasm is also a major impediment to clinical translation [7-9]. To avoid these drawbacks, the ideal siRNA delivery system should efficiently encapsulate the negatively charged siRNA molecule, prevent degradation by endogenous enzymes, and facilitate cellular uptake and intracellular release. 
Technologies already in clinical trials addressing the delivery of RNAi therapeutics will be presented in the following sections. The last section will highlight some examples of current trends and novel applications of nanomedicine in the field of combination therapy.

\section{Methods of nanoparticle preparation}

Their nano-scale size means that NPs require a very specialized formulation method. The most common methods employ self-assembly processes to amphiphilic lipid, polymer, or polymerdrug conjugates. Such processes include nanoprecipitation, oil-in-water $(\mathrm{O} / \mathrm{W})$ single emulsion, and water-in-oil-in-water (W/O/W) double emulsification [10-12]. The most recent development in the synthesis of NPs involves the discipline of microfluidics, which is capable of manipulating nano-scale volumes in micro-scale fluidic channels [13]. Microfluidic reactors offer precise control and manipulation of the fluids used to create NPs. Micro-scale channels offer the advantage of a very large surface-to-volume ratio and controllable mixing time, which promotes higher NP yield and uniform size [14,15]. Through multi-inlet mixing at different ratios and hydrodynamic flow focusing, the NPs self-assemble through diffusive mass transfer at the interface of miscible liquids (Figure 1) [12]. Other significant advantages of microfluidics include the reproduciblility of device fabrication and rapid, consistent NP synthesis with narrow size distributions [14]. Microfluidic devices are tunable and can use 3D hydrodynamic focusing to create NPs of different sizes and targeting ligand densities with multiple polymers, which can in turn produce diverse NP libraries (Figure 1) [16,17]. In addition, microfluidics provides a means to rapidly and continuously form consistent nano- and micro-structures while simultaneously encapsulating drugs, which is not readily feasible with conventional approaches $[18,19]$. However, to take full advantage of the benefits of microfluidic nanoformulation, the challenges associated with the high costs of glass/silicon fabrication and large-scale production for clinical use still need to be addressed [14].

\section{"Stealth" modification of NPs}

Modification with PEG is currently the gold standard for NP coating [10,20,21]. PEG surface functionalization has been shown to dramatically reduce protein adsorption, particularly apolipoprotein $\mathrm{J}$ and complement protein $\mathrm{C} 3$, through hydrophilicity and steric repulsion effects, with the effect of extending circulation time in blood [22-24]. This has allowed the "stealth" NP carriers to persist in the bloodstream long enough to reach or recognize their therapeutic site of action [25]. Examples of stealth nanocarriers include PEGylated liposomal doxorubicin (Doxil ${ }^{\circledR}$ ) and the PLA-PEG micelle form of paclitaxel (Genexol-PM®). Since the first PEGylated nanomedicine, Doxil@, was approved in 1995, many of the current FDA-approved NPs and NPs in clinical trials have begun to carry the PEG modification. In addition to PEGylation, new biomaterials and delivery strategies have been developed to prolong the circulation time of NPs [26-29]. For example, zwitterionic polymer-based NPs are resistant to non-specific protein adsorption, due to electrostatically induced hydration [30,31]. Modification of the zwitterionic polymer with a pH-switchable moiety allows the NP surface charge to be altered and recognizable by tumor cells, based on $\mathrm{pH}$ differences between normal tissue and the tumor microenvironment [31]. The switchable 
surface charge of these particles allows more efficient cellular uptake than the highly hydrophilic PEG NP [26,27,31].

One concept prevalent in scientific innovation is the notion of borrowing from nature. The longest circulation time achieved by synthetic particles in clinical trials is under 300 hours, whereas the human red blood cell circulates for 100-120 days in the body [32]. This is mainly because the membrane protein CD47, a "self-marker" on cell membranes (including red blood cells of humans, mice, and other mammals), signals the phagocyte receptor CD172a, preventing cells from being phagocytosed [33]. NPs modified with a synthetic minimal "self" peptide that was computationally designed based on human CD47 showed prolonged circulation half-life in a mouse model [33]. Further research extended this concept to prolong the residence time of NPs in vivo by coating PLGA NPs with erythrocyte membranes that incorporated the mouse's own membrane lipids and membrane proteins (Figure 2). Preliminary preclinical tests showed that these novel NPs had a longer circulation half-life than PEG-coated NPs [34]. Although more research is necessary, zwitterionic and erythrocyte-coated NPs may become viable PEG substitutes, as zwitterions offer increased uptake, and erythrocytes pose little risk of immunogenicity from the patients' own somatic cells.

\section{Nanoformulation and controlled release}

Nanoformulation is an important opportunity to revisit promising molecular entities that failed in the development process due to poor pharmaceutical properties such as high cytotoxicity or poor cellular uptake. A recent example is CRLX101, which is a polymerbased NP containing Camptothecin (CPT) conjugated to a cyclodextrin-containing polymer (CDP) for the treatment of solid tumors [35]. Unfavorable cytotoxic effects led to the shelving of development of CPT in spite of clear efficacy in tumor suppression. The CRLX101 NP displays a sustained intracellular release profile, which lowers systemic exposure and significantly decreases CPT toxicity [35]. The phase I/IIa study shows low levels of toxicity and promising anti-tumor activity [35]. CRLX101 partly solves the decades-long problem of CPT toxicity by using NPs to release a controlled amount of CPT over a longer period of time. Many promising drugs such as camptothecin and wortmannin failed clinical development because they did not meet toxicity, stability, or solubility requirements. Nanomedicine has the potential to solve these problems and revive abandoned cancer drugs for clinical use [36].

\section{Passive targeting}

A major benefit of nanomedicine is the improved biodistribution of therapeutic agents through passive targeting, a defining feature of first-generation NPs. The enhanced permeability and retention (EPR) effect refers to the fact that tumors retain more polymeric NPs, proteins, liposomes, and micelles than other tissues [10,37,38]. Most tumors have an abnormally dense and permeable vasculature created through stimulation by vascular endothelial growth factor (VEGF). Tight junctions in normal vasculature prevent particles larger than $2 \mathrm{~nm}$ from crossing between endothelial cells. However, the tight junctions and basement membrane of tumor vasculature are disordered, allowing entities from 10-500 nm 
in size to extravasate and accumulate within the tumor interstitium $[39,40]$. The lymphatic drainage system is also impaired in tumors, further entrapping macromolecular particles and delaying their clearance [41,42]. Passive targeting is based on both the minute size of drug carriers as well as the leaky neovasculature of the tumor (Figure 3). With the longer blood circulation brought about by "stealth" modification (e.g. PEGylation), increased accumulation of NPs is possible through the EPR effect [39].

Though the notion of utilizing NPs for therapeutic purposes has existed for decades, nanotherapeutics that have reached the market have met with varying degrees of success [43]. Doxil ${ }^{\circledR}$ was approved for clinical use in 1995 to treat AIDS-related Kaposi's sarcoma, ovarian cancer, and other cancers [44]. Encapsulating doxorubicin within PEGylated NPs allows for extended circulation half-life and higher tumor concentration of the drug. Homing to the disease site is driven solely by the particles' nano-dimensions through the EPR effect [45] rather than any specific recognition of the target. Another example of a passivetargeting nanomedicine is Genexol-PM®, a polymeric micelle delivery system whose formulation includes poly(D,L-lactide), which allows for controlled release of therapeutic (Genexol-PM® was approved in Korea in 2007).

ALN-TTR02 is a lipid nanoparticle (LNP) that encapsulates siRNA targeting a conserved sequence in the 3' untranslated region of the transthyretin (TTR) gene. The NP structure consists of a neutral lipid, a PEG lipid, and an ionizable cationic lipid to facilitate encapsulation of negatively charged siRNA through electrostatic interactions [46]. It is used to treat transthyretin amyloidosis, a condition produced by a mutant TTR gene that causes the accumulation of transthyretin amyloid in peripheral nerves and the heart [47]. Phase II trials showed higher knockdown and continuing suppression of TTR with varying single doses as compared to placebo. The therapy seems to be generally safe, with no serious adverse events yet reported [47]. However, ALN-TTR02 targets delivery to the liver, which is already a proven site of NP accumulation due to reticuloendothelial system uptake. There is still progress to be made to treat diseases requiring the differential biodistribution of therapeutics.

TKM-PLK1 is another lipid nanoparticle similar in structure to ALN-TTR02, encapsulating siRNA that inhibits the protein product polo-like kinase 1 (PLK1). PLK1 phosphorylates Cdc25C, regulates DNA damage checkpoints, microtubule nucleation, chromosomal condensation and segregation, and is an important target for therapeutic treatment [48]. This nanoformulation is significant because it does not rely on accumulation in the organs of the reticuloendothelial system (such as the liver) to deliver its payload. TKM-PLK1 relies mainly on the EPR effect to localize NPs into solid tumors, with encouraging results [49]. Phase I trials measured the effects of dose escalation on solid tumors in advanced cancer patients with promising safety and efficacy results, culminating in an ongoing phase II clinical trial for patients with advanced Gastrointestinal Neuroendocrine Tumors (GI-NET) or Adrenocortical Carcinoma (ACC).

NP therapeutics in clinical trials are clearly an improvement over current treatments. ALNTTR02 uses gene silencing to knock out mutant TTR production. This shows promise as a viable alternative to invasive procedures for TTR-mediated amyloidosis such as liver 
transplantation, as well as offering a possible co-treatment with TTR stabilizers such as diflunisal. TKM-PLK1 is delivered systemically to solid tumors, and the phase I trials targeting PLK-1 for tumour proliferation have had generally positive results. Due to the specific sizes of the NPs described above, the EPR effect combined with hemodynamic and diffusive mechanisms contributes to the longer blood circulation and accumulation of NPs in the tumor. However, passive targeting has a number of drawbacks. Suboptimal biodistribution, with particles being trapped mainly in the liver and spleen due to reticuloendothelial function, is a major impediment to efficient delivery. In addition, the extent of the EPR effect varies from tumor to tumor and even intratumorally, due to the heterogeneity and vascular permeability differences within an individual tumor [40,50]. Furthermore, the higher interstitial pressure within the tumor core causes the NPs to flow from the inner regions to the outer regions, further exacerbating this issue [10]. Two major challenges must be resolved in targeted delivery: further extending blood circulation times and homing the NPs towards specific sites of targeting for intracellular delivery. Therefore efforts are needed to synergize passive targeting with a more dynamic method capable of further improving accumulation of NPs at disease sites.

\section{Active targeting nanomedicine}

Even with the improvements in biodistribution offered by the EPR effect and PEGylation, the majority of a therapeutic (upwards of $90 \%$ ) will inevitably be concentrated in the reticuloendothelial organs such as the liver and spleen due to clearance by mononuclear phagocytes [51]. Active targeting is being explored as a method to achieve spatial localization by intentionally homing NPs to active diseased sites while eliminating off-target adverse effects in normal tissue. The polyvalent decoration of a NP's surface with a ligand can facilitate binding to a biomarker specifically overrepresented in targeted cells and trigger receptor-mediated endocytosis (Figure 3), a process that has considerable implications for targeted delivery [5]. The ligands used to modify NPs include antibodies (Figure 4), engineered antibody fragments, proteins, peptides, small molecules, and aptamers [52]. The specific ligand-receptor interaction can be utilized to concentrate a therapeutic nanomedicine at a diseased tissue in vivo, producing a preferred distribution profile $[3,53]$. The ligand density can be fine-tuned in the formulation process to optimize avidity $[10,54]$.

A few active targeting nanoplatforms making use of ligand-receptor interactions have made their way to clinical trials. The first targeted NP delivery system to feature siRNA was CALAA-01, which consists of a cyclodextrin-containing cationic polymer, a PEG corona, and human transferrin (Tf) as a targeting ligand [55]. The Tf on the nanoparticle surface binds to overexpressed transferrin receptors (TfR) on cancer cells, and the nanoparticles are then internalized via receptor-mediated endocytosis. These siRNA-containing targeted nanoparticles were administered intravenously to melanoma patients, upon which they circulated in the body and localized in tumors $[55,56]$. Tumor biopsies showed a correlation between the dose administered and the amounts of intracellularly localized NPs. Furthermore, levels of both the specific messenger RNA and the protein were lower after injection of the targeted nanoparticles. In this study, TfR was used as a potent target; it is typically upregulated on cancer cells and triggers cellular uptake via clathrin-coated pits. It 
is also ubiquitously expressed in all types of tissues to satisfy the iron requirements of dividing cells. Other examples of ubiquitous receptors, including folate receptors and the receptor tyrosine kinase EGFR, have been explored for active targeting delivery of nanotherapeutics to tumor cells [57-59].

Biomarkers distinctively expressed by certain organs offer the possibility to further improve the specificity of nanomedicine treatments. Prostate-specific membrane antigen (PSMA) is a good example of such a tissue-specific receptor. BIND-014, a targeted nanomedicine functionalized with the PSMA-specific ligand, is currently in human clinical trials. It is a polymeric nanoparticle encapsulating docetaxel (DTXL) for solid tumor treatment. This nanoparticle is modified with a PSMA substrate analog inhibitor, S,S-2-[3-[5-amino-1carboxypentyl]-ureido]-pentanedioic acid (ACUPA), specific to PSMA, which is upregulated on prostate cancer cells. In Phase I clinical trials, BIND-014 displays promising results in patients with advanced or metastatic large tumors [1]. Encouragingly, tumor recession has been observed in patients with cancer unresponsive to other treatments [60]. The enhanced therapeutic index of DTXL was mainly attributed to PSMA targeting, which is consistent with preclinical results. Phase II clinical trials of BIND-014 are under investigation for treatment of metastatic drug-resistant prostate cancer and non-small cell lung cancer. Other specific targets have been investigated for targeted drug delivery. For example, the increasing availability of monoclonal antibodies has fostered interest in antibody-functionalized nanomedicines for many years [61,62].

Active targeting with nanoparticles has yielded promising findings in preclinical studies and, in some cases, early clinical trials. On the other hand, some studies involving nanoparticle targeting have been inconclusive, and therapeutic efficacy in humans has not yet been convincingly demonstrated overall $[3,51,63]$. The protein corona that forms around an NP as it interacts with physiological proteins in the body as well as factors that interrupt the orientation and proper display of the targeting ligand are two variables that highlight the need for further studies of the clinical relevance of actively targeted nanomedicine $[3,63]$.

\section{Nanoparticle-based Combination Therapy}

Cancers are very complex diseases involving multiple pathways, and their progression is marked by many successive mutations in a line of cells. In addition, since mutations favorable to the survival of tumor cells are selected as chemotherapy progresses [64], tumors often present such challenges as intrinsic and acquired resistance to chemotherapeutics. Therefore the inhibition of a pathway by a single drug may not be sufficient to bring about tumor recession. In combination chemotherapy, the synergistic effect of two (or more) agents targeting different disease pathways, genes, or cell-cycle checkpoints in the cancer process are leveraged to raise the chances of eliminating cancer (Figure 3). The combination of chemotherapy medications allows oncologists to use drugs at lower doses, reducing cytotoxic effects but increasing efficacy, and therefore present a promising approach for cancer research [10]. In practice, combination chemotherapy results in a better response and improved survival compared with single-agent therapy; recent examples include the combination of Platinol (cisplatin) and Navelbine (vinorelbine) to treat non-small cell lung 
cancer and TCH (Taxol, Carboplatin and Herceptin) for treatment of HER2/neu-positive tumors $[65,66]$.

Nevertheless, the effective administration of multiple drugs at an optimized dosage ratio is complicated by dissimilar pharmacokinetics and biodistribution, due to different rates of metabolism within the body [67]. Nanoformulations can help avoid such limitations by carrying (in one NP) multiple therapeutic agents with different physicochemical properties and pharmacological behaviors. In addition, NPs are able to maintain the optimized synergistic drug ratio in a single carrier up to the point of intracellular uptake to the target cancer cell. This ratio may not be maintained by the use of separate carriers, which each encapsulate a different drug. Currently this novel "two-in-one" approach is under clinical and preclinical investigation.

CPX-351 is a liposomal NP for the treatment of acute myeloid leukemia designed to incorporate the chemotherapy drugs cytarabine and daunorubicin in an optimized 5:1 molar ratio. Such a combination has previously been used clinically with small-molecule drugs, but the efficacy was limited by unsuitable pharmacokinetics and poor solubility, requiring co-administration with toxic solvents [68]. In phase I and II trials, CPX-351 increased overall survival in first-relapse patients [69] and is currently in phase III clinical trials. Other combinations such as CPX-1 (irinotecan/floxuridine) and paclitaxel/tanespimycin are under preclinical/clinical investigation $[68,70,71]$.

Combinatorial therapies involving both siRNA and miRNA have the potential benefit of dual inhibition of a target gene product, as well as modulation of oncogenes within the same pathway. A team used univariate Cox regression analysis and multiple miRNA target prediction programs on a dataset from a previous ovarian cancer study to identify miRNA candidates likely to improve antitumor potency when combined with EphA2 targeting siRNA [72]. EphA2 is overexpressed in ovarian cancer and is associated with shorter median survival [73]. In a mouse model, the chosen miRNA:siRNA combination was delivered via a neutral liposomal nanocarrier composed of 1,2-dioleoyl-sn-glycero-3phosphatidylcholine (DOPC) and demonstrated tumorsuppressive effect superior to either miRNA or siRNA alone [72]. In addition, a multiple siRNA combination therapy recently demonstrated increased efficacy in phase I clinical trials [74]. The combination of siRNA and miRNA technologies is a significant step toward realizing the full potential of RNAi therapies.

The combination of chemotherapy with RNAi is also a promising synergistic strategy for cancer treatment. Recently, a polymeric NP platform composed of an aqueous inner core, a cationic and hydrophobic PLGA layer, and a hydrophilic PEG corona was developed to circumvent acquired chemoresistance by simultaneously delivering a cisplatin prodrug and REV1/REV3L-specific siRNAs, which suppress gene targets crucial to translesion synthesis (TLS) pathways in tumors [75]. Most mutations that result from DNA damage are the consequence of error-prone TLS DNA synthesis, which plays a significant role in cisplatininduced mutations. This eventually results in acquired chemoresistance by improving the capacity of tumor cells to either repair or tolerate DNA damage [76,77]. NPs were shown to synergistically suppress the target genes involved in TLS, resulting in tumor cell 
sensitization to chemotherapeutic and tumor inhibition in a mouse model that was more effective than cisplatin monotherapy. Though this smallmolecule drug/siRNA approach is still far from clinical evaluation, it presents a robust platform that not only screens and validates target pathways involved in drug resistance, but also achieves an efficacy that may not be possible with dual-drug or RNAi combinations alone.

\section{Concluding Remarks and Future Perspectives}

In conventional oral or intravenous drug delivery of small-molecule drugs, the medicine is distributed indiscriminately throughout the body, with arbitrary concentrations reaching both the disease site and healthy tissue. Chemotherapeutic agents in general cause unintended adverse effects to healthy tissue and require a trade-off between optimal disease treatment and patient quality of life. Nanoparticle-based drug delivery systems offer revolutionary opportunities to develop highly effective targeted therapeutics with improved circulation half-life, bioavailability, biodistribution, pharmacokinetics, and safety profiles. In addition, nanoparticles are indispensable in maintaining synergistic drug ratios in combinational therapy and offer the first possibility of delivering therapeutic agents such as nucleic acids and unstable proteins. The codelivery of adjuvants with antigens to tumors promotes antigen-specific immune responses against the cancer and is yet another facet of the numerous nanoparticle anticancer therapies in development [78]. However, there is still much to be learned in the emerging field of nanomedicine. We have yet to develop a carrier that can effectively deliver a payload intratumorally with clinically validated results. Extending circulation closer to the time scale of red blood cells and retention of particles at the disease site rather than the reticuloendothelial organs remain significant challenges.

Advances in nanomedicine occur through the development of novel nanocarriers and technologies for drug delivery. An ideal nanocarrier should fit the following profile: (i) biodegradable and biocompatible; (ii) capable of effective homing, with the majority of therapeutics localized within the target site; (iii) designed with optimal biophysicochemical properties for superior drug loading, circulation half-life, and sustained drug release across infrequent administration times; and (iv) amenable to cost-effective scale-up for commercialization. The refinement and incorporation of these qualities in one nanocarrier is the "holy grail" of nanomedicine, synthesizing cutting-edge knowledge and technologies from the disciplines of medicine, chemistry, engineering, and physics.

Besides the complications in the experimental design of nanoparticles, there exist multiple challenges in the manufacturing, regulation, and approval of NPs for clinical use.

Compliance with quality-control guidelines such as good laboratory quality practice (GLP), good manufacturing practice (GMP), as well as passing the three phases of FDA Investigational New Drug trials will be challenging in bringing a new nanoformulation to market [6]. Moreover, patent disputes are becoming more frequent as more companies acquire broad patent rights on a wide range of nanoparticle compositions and usage methods [79].

Yet these challenges in nanomedicine are accompanied by new opportunities as well. The field of cancer nanomedicine has begun to experience success in clinical applications and 
has yet to reach its full potential. There is mounting evidence that effective encapsulation of small-molecule drugs, nucleic acids, or other compounds may be capable of mediating comprehensive cancer management, or even a potential cure [6]. The virtually limitless modular possibilities for different ligands, materials, and therapeutic nanoformulations coupled with improved treatment efficacies allow us to consider NPs not just drug delivery vehicles, but an entirely new class of therapeutics [10]. The broad range of diseases NPs are capable of treating, the considerable amount of important research yet to be carried out, and the potential to commercialize novel formulations are undoubtedly important "draws" for the brightest minds in research. There is still "plenty of room at the bottom". The era of nanomedicine is poised to mature in the next few decades; incorporating elements of personalized medicine, it will affect the therapeutic world in a powerful and permanent way.

\section{Acknowledgements}

This work was supported by the National Institutes of Health (NIH) (grant CA151884), the National Heart, Lung, and Blood Institute, (NIH), through a Program of Excellence in Nanotechnology (PEN) Award, (Contract \#HHSN268201000045C) and the David Koch-Prostate Cancer Foundation Award in Nanotherapeutics. Dr. Xiaoyang Xu acknowledges postdoctoral support from an NIH National Research Service Award (NRSA) 1F32CA168163-03. Dr. Nicolas Bertrand acknowledges a post-doctoral fellowship from the Canadian Institutes of Health Research (CIHR). Dr. Farokhzad declares financial interests in BIND Biosciences, Selecta Biosciences, and Blend Therapeutics.

\section{References}

1. Sanna V, et al. Targeted therapy using nanotechnology: focus on cancer. Int. J. Nanomedicine. 2014; 9:467-483. [PubMed: 24531078]

2. Farokhzad OC, Langer R. Impact of nanotechnology on drug delivery. ACS nano. 2009; 3:16-20. [PubMed: 19206243]

3. Chrastina A, et al. Overcoming in vivo barriers to targeted nanodelivery. Wiley Interdiscip. Rev. Nanomed. Nanobiotechnol. 2011; 3:421-437. [PubMed: 21538941]

4. Davis ME, et al. Nanoparticle therapeutics: an emerging treatment modality for cancer. Nat. Rev. Drug Discov. 2008; 7:771-782. [PubMed: 18758474]

5. Zhang XQ, et al. Interactions of nanomaterials and biological systems: Implications to personalized nanomedicine. Adv. Drug Deliv. Rev. 2012; 64:1363-1384. [PubMed: 22917779]

6. Chow EKH, Ho D. Cancer Nanomedicine: From Drug Delivery to Imaging. Sci. Transl. Med. 2013:5.

7. Whitehead KA, et al. Knocking down barriers: advances in siRNA delivery. Nat. Rev. Drug Discov. 2009; 8:129-138. [PubMed: 19180106]

8. Shi J, et al. Differentially charged hollow core/shell lipid-polymer-lipid hybrid nanoparticles for small interfering RNA delivery. Angew. Chem. 2011; 50:7027-7031. [PubMed: 21698724]

9. Reynolds A, et al. Induction of the interferon response by siRNA is cell type- and duplex lengthdependent. Rna. 2006; 12:988-993. [PubMed: 16611941]

10. Kamaly N, et al. Targeted polymeric therapeutic nanoparticles: design, development and clinical translation. Chem. Soc. Rev. 2012; 41:2971-3010. [PubMed: 22388185]

11. Rao JP, Geckeler KE. Polymer nanoparticles: Preparation techniques and size-control parameters. Prog. Polym. Sci. 2011; 36:887-913.

12. Capretto L, et al. Microfluidic and lab-on-a-chip preparation routes for organic nanoparticles and vesicular systems for nanomedicine applications. Adv. Drug Deliv. Rev. 2013; 65:1496-1532. [PubMed: 23933616]

13. Whitesides GM. The origins and the future of microfluidics. Nature. 2006; 442:368-373. [PubMed: 16871203] 
14. Valencia PM, et al. Microfluidic technologies for accelerating the clinical translation of nanoparticles. Nat. Nanotechnol. 2012; 7:623-629. [PubMed: 23042546]

15. Lim JM, et al. Ultra-high throughput synthesis of nanoparticles with homogeneous size distribution using a coaxial turbulent jet mixer. ACS nano. 2014; 8:6056-6065. [PubMed: 24824296]

16. Valencia PM, et al. Microfluidic platform for combinatorial synthesis and optimization of targeted nanoparticles for cancer therapy. ACS nano. 2013; 7:10671-10680. [PubMed: 24215426]

17. Lim JM, et al. Parallel microfluidic synthesis of size-tunable polymeric nanoparticles using 3D flow focusing towards in vivo study. Nanomedicine. 2014; 10:401-409. [PubMed: 23969105]

18. Windbergs M, et al. Biodegradable core-shell carriers for simultaneous encapsulation of synergistic actives. J. Am. Chem. Soc. 2013; 135:7933-7937. [PubMed: 23631388]

19. Choi C-H, et al. One Step Formation of Controllable Complex Emulsions: From Functional Particles to Simultaneous Encapsulation of Hydrophilic and Hydrophobic Agents into Desired Position (Adv. Mater. 18/2013). Adv. Mater. 2013; 25:2535-2535.

20. Harris JM, Chess RB. Effect of pegylation on pharmaceuticals. Nat. Rev. Drug Discov. 2003; 2:214-221. [PubMed: 12612647]

21. Jokerst JV, et al. Nanoparticle PEGylation for imaging and therapy. Nanomedicine. 2011; 6:715728. [PubMed: 21718180]

22. Gref R, et al. 'Stealth' corona-core nanoparticles surface modified by polyethylene glycol (PEG): influences of the corona (PEG chain length and surface density) and of the core composition on phagocytic uptake and plasma protein adsorption. Colloids Surf., B. 2000; 18:301-313.

23. Peracchia MT, et al. Visualization of in vitro protein-rejecting properties of PEGylated stealth ${ }^{\circledR}$ polycyanoacrylate nanoparticles. Biomaterials. 1999; 20:1269-1275. [PubMed: 10403044]

24. Bazile D, et al. Stealth Me.PEG-PLA nanoparticles avoid uptake by the mononuclear phagocytes system. J. Pharm. Sci. 1995; 84:493-498. [PubMed: 7629743]

25. Peracchia MT, et al. Stealth ${ }^{\circledR}$ PEGylated polycyanoacrylate nanoparticles for intravenous administration and splenic targeting. J. Control. Release. 1999; 60:121-128. [PubMed: 10370176]

26. Radovic-Moreno AF, et al. Surface charge-switching polymeric nanoparticles for bacterial cell wall-targeted delivery of antibiotics. ACS nano. 2012; 6:4279-4287. [PubMed: 22471841]

27. Hatakeyama $\mathrm{H}$, et al. A multifunctional envelope type nano device (MEND) for gene delivery to tumours based on the EPR effect: a strategy for overcoming the PEG dilemma. Adv. Drug Deliv. Rev. 2011; 63:152-160. [PubMed: 20840859]

28. Yang W, et al. Poly(carboxybetaine) nanomaterials enable long circulation and prevent polymerspecific antibody production. Nano Today. 2014; 9:10-16.

29. Keefe AJ, Jiang S. Poly(zwitterionic)protein conjugates offer increased stability without sacrificing binding affinity or bioactivity. Nat. Chem. 2012; 4:59-63. [PubMed: 22169873]

30. Zhu Y. A robust graft-to strategy to form multifunctional and stealth zwitterionic polymer-coated mesoporous silica nanoparticles. Biomacromolecules. 2014; 15:1845-1851. [PubMed: 24670217]

31. Yuan YY, et al. Surface charge switchable nanoparticles based on zwitterionic polymer for enhanced drug delivery to tumor. Adv. Mater. 2012; 24:5476-5480. [PubMed: 22886872]

32. Alexis F, et al. Nanoparticle technologies for cancer therapy. Handb. Exp. Pharmacol. 2010:55-86. [PubMed: 20217526]

33. Rodriguez PL, et al. Minimal "Self" peptides that inhibit phagocytic clearance and enhance delivery of nanoparticles. Science. 2013; 339:971-975. [PubMed: 23430657]

34. Hu CM, et al. Erythrocyte membrane-camouflaged polymeric nanoparticles as a biomimetic delivery platform. Proc. Natl. Acad. Sci. U.S.A. 2011; 108:10980-10985. [PubMed: 21690347]

35. Weiss GJ, et al. First-in-human phase 1/2a trial of CRLX101, a cyclodextrin-containing polymercamptothecin nanopharmaceutical in patients with advanced solid tumor malignancies. Invest. New Drugs. 2013; 31:986-1000. [PubMed: 23397498]

36. Karve $S$, et al. Revival of the abandoned therapeutic wortmannin by nanoparticle drug delivery. Proc. Natl. Acad. Sci. U.S.A. 2012; 109:8230-8235. [PubMed: 22547809]

37. Acharya S, Sahoo SK. PLGA nanoparticles containing various anticancer agents and tumour delivery by EPR effect. Adv. Drug Deliv. Rev. 2011; 63:170-183. [PubMed: 20965219] 
38. Maeda H, et al. Polymeric drugs for efficient tumor-targeted drug delivery based on EPR-effect. Eur. J. Pharm. Biopharm. 2009; 71:409-419. [PubMed: 19070661]

39. Torchilin V. Tumor delivery of macromolecular drugs based on the EPR effect. Adv. Drug Deliv. Rev. 2011; 63:131-135. [PubMed: 20304019]

40. Bertrand N, et al. Cancer nanotechnology: the impact of passive and active targeting in the era of modern cancer biology. Adv. Drug Deliv. Rev. 2014; 66:2-25. [PubMed: 24270007]

41. Fang J, et al. The EPR effect: Unique features of tumor blood vessels for drug delivery, factors involved, and limitations and augmentation of the effect. Adv. Drug Deliv. Rev. 2011; 63:136151. [PubMed: 20441782]

42. Maeda H, et al. The EPR effect for macromolecular drug delivery to solid tumors: Improvement of tumor uptake, lowering of systemic toxicity, and distinct tumor imaging in vivo. Adv. Drug Deliv. Rev. 2013; 65:71-79. [PubMed: 23088862]

43. Venditto VJ, Szoka FC. Cancer nanomedicines: So many papers and so few drugs! Adv. Drug Deliv. Rev. 2013; 65:80-88. [PubMed: 23036224]

44. Barenholz Y. Doxil(R)--the first FDA-approved nano-drug: lessons learned. J. Control. Release. 2012; 160:117-134. [PubMed: 22484195]

45. Matsumura YMH. A new concept for macromolecular therapeutics in cancer chemotherapy: mechanism of tumoritropic accumulation of proteins and the antitumor agent SMANCS doi. Cancer Res. 1986; 46:6387-6392. [PubMed: 2946403]

46. Semple SC, et al. Rational design of cationic lipids for siRNA delivery. Nat. biotechnol. 2010; 28:172-176. [PubMed: 20081866]

47. Coelho T, et al. Safety and efficacy of RNAi therapy for transthyretin amyloidosis. N. Engl. J. Med. 2013; 369:819-829. [PubMed: 23984729]

48. Shi W, et al. Significance of Plk1 regulation by miR-100 in human nasopharyngeal cancer. Int. J. Cancer. 2010; 126:2036-2048. [PubMed: 19739117]

49. Wan C. Lipid nanoparticle delivery systems for siRNA-based therapeutics. Drug Deliv. Transl. Res. 2013; 4:74-83. [PubMed: 25786618]

50. Danquah MK, et al. Extravasation of polymeric nanomedicines across tumor vasculature. Adv. Drug Deliv. Rev. 2011; 63:623-639. [PubMed: 21144874]

51. Albanese A, et al. The effect of nanoparticle size, shape, and surface chemistry on biological systems. Annu. Rev. Biomed. Eng. 2012; 14:1-16. [PubMed: 22524388]

52. Peer D, et al. Nanocarriers as an emerging platform for cancer therapy. Nat. Nanotechnol. 2007; 2:751-760. [PubMed: 18654426]

53. Ge Z, Liu S. Functional block copolymer assemblies responsive to tumor and intracellular microenvironments for site-specific drug delivery and enhanced imaging performance. Chem. Soc. Rev. 2013; 42:7289-7325. [PubMed: 23549663]

54. Valencia PM, et al. Effects of ligands with different water solubilities on self-assembly and properties of targeted nanoparticles. Biomaterials. 2011; 32:6226-6233. [PubMed: 21658757]

55. Davis ME. The first targeted delivery of siRNA in humans via a self-assembling, cyclodextrin polymer-based nanoparticle: from concept to clinic. Mol. Pharm. 2009; 6:659-668. [PubMed: 19267452]

56. Davis ME, et al. Evidence of RNAi in humans from systemically administered siRNA via targeted nanoparticles. Nature. 2010; 464:1067-1070. [PubMed: 20305636]

57. Zhang XQ, et al. Multimodal nanodiamond drug delivery carriers for selective targeting, imaging, and enhanced chemotherapeutic efficacy. Adv. Mater. 2011; 23:4770-4775. [PubMed: 21932280]

58. Leamon CP, Low PS. Delivery of macromolecules into living cells: a method that exploits folate receptor endocytosis. Proc. Natl. Acad. Sci. 1991; 88:5572-5576. [PubMed: 2062838]

59. Sega EI, Low PS. Tumor detection using folate receptor-targeted imaging agents. Cancer Metast. Rev. 2008; 27:655-664.

60. Hrkach J, et al. Preclinical development and clinical translation of a PSMA-targeted docetaxel nanoparticle with a differentiated pharmacological profile. Science translational medicine. 2012; 4:128ra139. 
61. Johnston AP, et al. Targeting cancer cells: controlling the binding and internalization of antibodyfunctionalized capsules. ACS nano. 2012; 6:6667-6674. [PubMed: 22872125]

62. Steichen SD, et al. A review of current nanoparticle and targeting moieties for the delivery of cancer therapeutics. Eur. J. Pharm. Sci. 2012; 48:416-427. [PubMed: 23262059]

63. Mahon E, et al. Designing the nanoparticle-biomolecule interface for "targeting and therapeutic delivery". J. Control. Release. 2012; 161:164-174. [PubMed: 22516097]

64. Iyer AK, et al. Role of integrated cancer nanomedicine in overcoming drug resistance. Adv. Drug Deliv. Rev. 2013; 65:1784-1802. [PubMed: 23880506]

65. Douillard J-Y, et al. Adjuvant vinorelbine plus cisplatin versus observation in patients with completely resected stage IB-IIIA non-small-cell lung cancer (Adjuvant Navelbine International Trialist Association [ANITA]): a randomised controlled trial. Lancet Oncol. 2006; 7:719-727. [PubMed: 16945766]

66. Robert N, et al. Randomized phase III study of trastuzumab, paclitaxel, and carboplatin compared with trastuzumab and paclitaxel in women with HER-2-overexpressing metastatic breast cancer. J. Clin. Oncol. 2006; 24:2786-2792. [PubMed: 16782917]

67. Valencia PM, et al. Synergistic cytotoxicity of irinotecan and cisplatin in dual-drug targeted polymeric nanoparticles. Nanomedicine. 2013; 8:687-698. [PubMed: 23075285]

68. Ma L, et al. Nanoparticles for combination drug therapy. ACS nano. 2013; 7:9518-9525. [PubMed: 24274814]

69. Feldman EJ, et al. First-in-man study of CPX-351: a liposomal carrier containing cytarabine and daunorubicin in a fixed 5:1 molar ratio for the treatment of relapsed and refractory acute myeloid leukemia. J. Clin. Oncol. 2011; 29:979-985. [PubMed: 21282541]

70. Batist G, et al. Safety, pharmacokinetics, and efficacy of CPX-1 liposome injection in patients with advanced solid tumors. Clin. Cancer Res. 2009; 15:692-700. [PubMed: 19147776]

71. Katragadda U, et al. Combined delivery of paclitaxel and tanespimycin via micellar nanocarriers: pharmacokinetics, efficacy and metabolomic analysis. PloS one. 2013; 8:e58619. [PubMed: 23505544]

72. Nishimura M, et al. Therapeutic synergy between microRNA and siRNA in ovarian cancer treatment. Cancer Discov. 2013; 3:1302-1315. [PubMed: 24002999]

73. Landen CN, et al. EphA2 as a target for ovarian cancer therapy. Expert Opin. Ther. Targets. 2005; 9:1179-1187. [PubMed: 16300469]

74. Tabernero J, et al. First-in-humans trial of an RNA interference therapeutic targeting VEGF and KSP in cancer patients with liver involvement. Cancer Discov. 2013; 3:406-417. [PubMed: 23358650]

75. Xu X XK, Zhang XQ, Pridgen EM, Park GY, Cui DS, Shi J, Wu J, Kantoff PW, Lippard SJ, Langer R, Walker GC, Farokhzad OC. Enhancing tumor cell response to chemotherapy through nanoparticle-mediated codelivery of siRNA and cisplatin prodrug. Proc. Natl. Acad. Sci. U.S.A. 2013; 110:18638-18643. [PubMed: 24167294]

76. Xie K, et al. Error-prone translesion synthesis mediates acquired chemoresistance. Proc. Natl. Acad. Sci. U.S.A. 2010; 107:20792-20797. [PubMed: 21068378]

77. Doles J, et al. Suppression of Rev3, the catalytic subunit of Pol\{zeta\}, sensitizes drug-resistant lung tumors to chemotherapy. Proc. Natl. Acad. Sci. U.S.A. 2010; 107:20786-20791. [PubMed: 21068376]

78. Krishnamachari Y, et al. Nanoparticle Delivery Systems in Cancer Vaccines. Pharm. Res. 2011; 28:215-236. [PubMed: 20721603]

79. Harris DHK, Bawa R, Cleveland JT, O'Neill S. Strategies for resolving patent disputes over nanoparticle drug delivery systems. NLB. 2004; 1:1-18. 


\section{Glossary}

Active targeting

\author{
Amphiphilic \\ Combinatorial \\ nanodelivery
}

Liposome

Microfluidics

Mononuclear

phagocyte system

(MPS)

Nanomedicine

Nanoparticle

Passive

targeting/EPR

Effect

PLGA

Polyethylene

glycol (PEG)

RNA interference

(RNAi)

Zwitterionic

polymer
The targeted homing of nanoparticles to sites of disease by way of modifying the surface of the particle with ligands specific to biomarkers overrepresented in target cells.

Possessing both hydrophilic and hydrophobic parts.

The delivery of more than a single therapeutic in one particle, often in an optimized ratio for synergistic effect. Multiple cancer pathways may be targeted with one particle.

A spherical vesicle composed of a lipid bilayer.

A technology used to quickly fabricate uniform nanoparticles by manipulating minute amounts of liquids via channels on the micrometer scale.

The MPS, also called the reticuloendothelial system, consists of the phagocytes located in reticular connective tissue present in the liver, lymph nodes and spleen which are responsible for the eventual clearance of the majority of nanoparticles.

The design and development of therapeutics and diagnostic tools, distinguished by the nanoscopic scale of its delivery vehicles and diagnostic agents.

Particles, usually composed of lipid or polymer, typically less than $200 \mathrm{~nm}$ in diameter.

Refers to the observation that the permeable vasculature and disordered basement membrane of tumor tissue leads to preferential accumulation of entities $10-500 \mathrm{~nm}$ in size.

Poly(D,L-lactide-co-glycolide) is a commonly used polymer for the construction of nanoparticles, usually selected for its controlled release capabilities.

PEG is a polymer used to modify the nanoparticle surface, resulting in the prevention of non-specific binding to blood components.

These "stealth" particles are better able to evade clearance by cells of the MPS.

RNAi is a pathway in eukaryotic cells where short pieces of RNA are able to induce the breakdown of the complementary mRNAs.

A polymer which is capable of exhibiting both positive and negative charges, and has been shown to resist non-specific protein adsorption. 


\section{Highlights}

Nanomedicine brings new solutions for cancer therapy

Nanomedicine has the potential to target specific cells for chemotherapeutic treatment

Stealth tech can enhance circulation half-life and controlled release of therapeutic

Drug/RNAi combinatorial strategies show promising synergistic effects. 


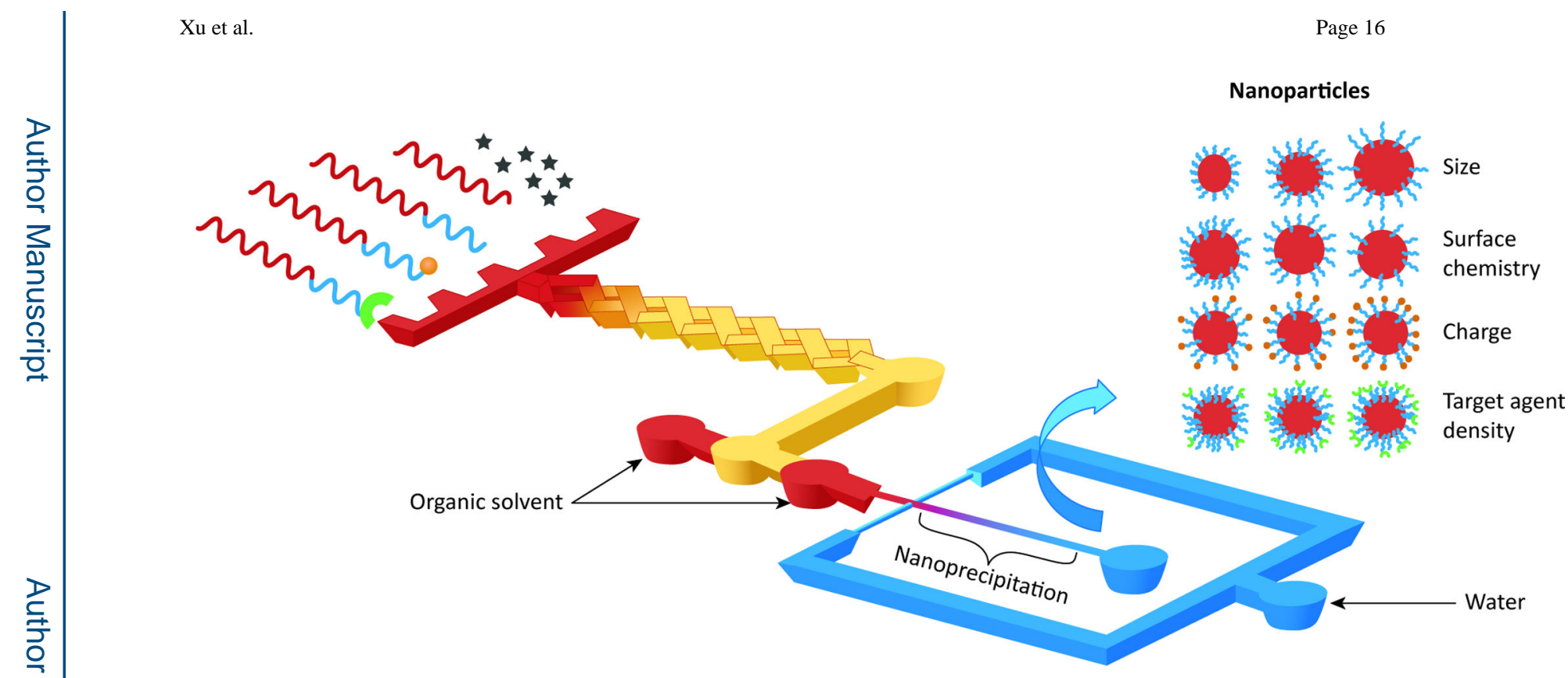

Figure 1. Schematic of a system in which nanoparticle (NP) precursors enter a multi-inlet mixer at different ratios to self-assemble a library of NPs

Programmable mixing of polymer precursors allows for synthesis of NPs with a wide range of sizes, surface chemistry, charge, and targeting agent densities. Adapted with permission from [16]. 


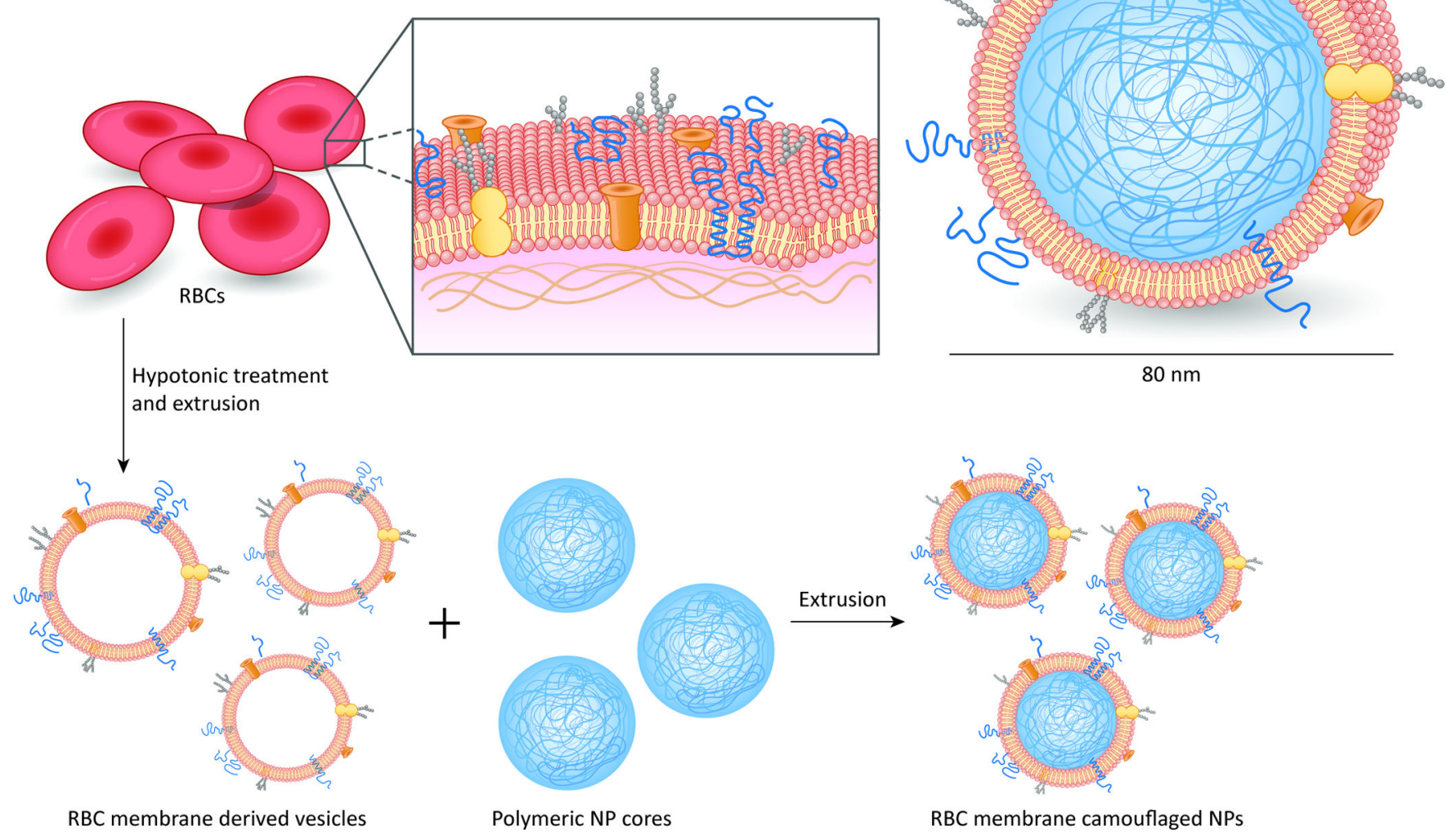

Figure 2. Red blood cell membrane-coated PLGA NPs

Cellular membranes provide a robust natural functionality to the particle. In comparative studies with PEG-coated NPs, RBC membrane-coated NPs exhibited a 39.6-hour half-life compared with 15.8 hours for PEG NPs. Adapted with permission from [34]. 


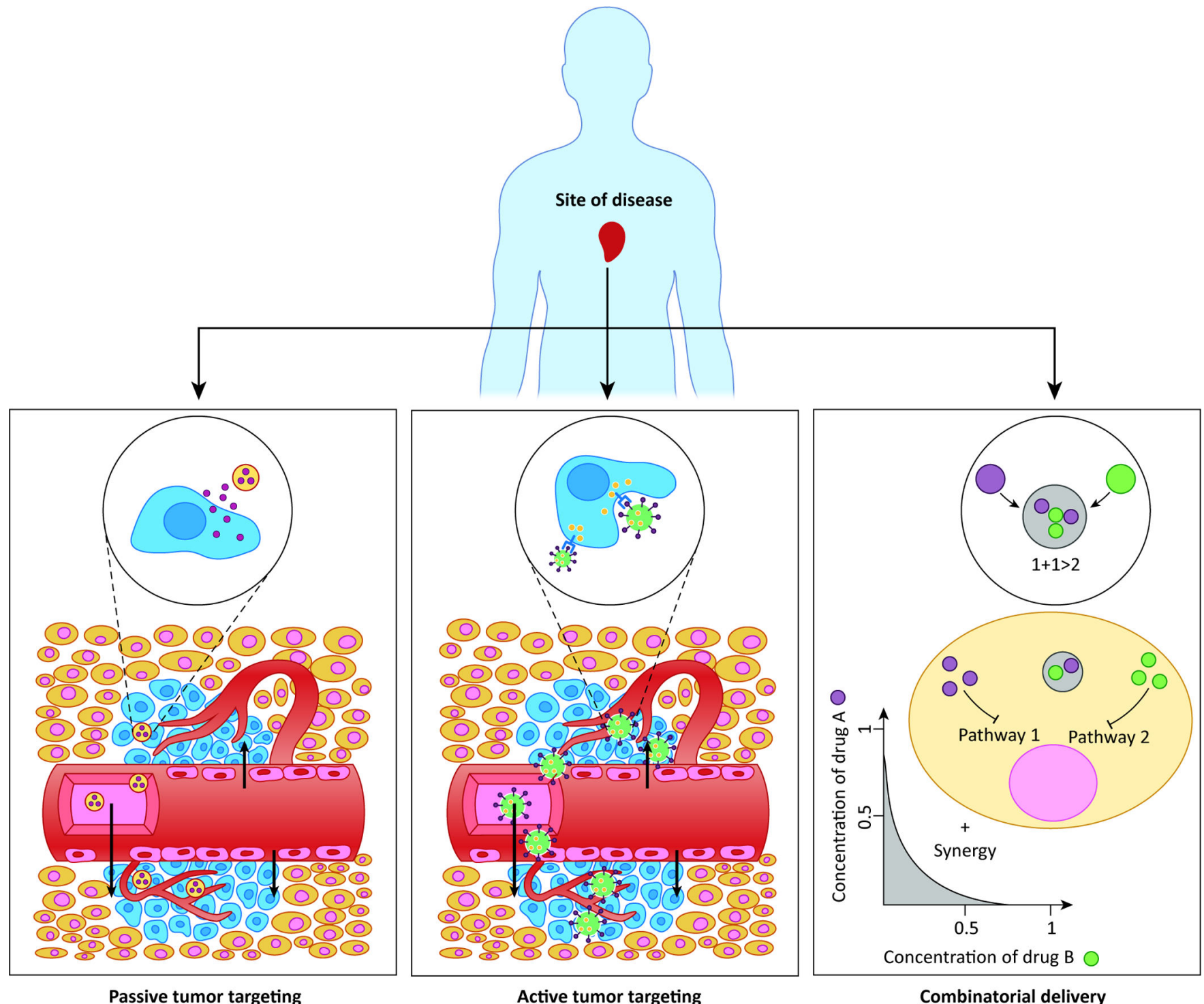

Figure 3. Passive targeting, active targeting, and combinatorial delivery

In passive targeting (left), the NPs passively extravasate though the leaky vasculature via the EPR effect and preferentially accumulate in tumors. In active targeting (middle), targeting ligands on the surface of the NP trigger receptor-mediated endocytosis for enhanced cellular uptake. In combinatorial delivery (right), two or more therapeutic agents inhibit different or identical disease pathways for a synergistic effect. 


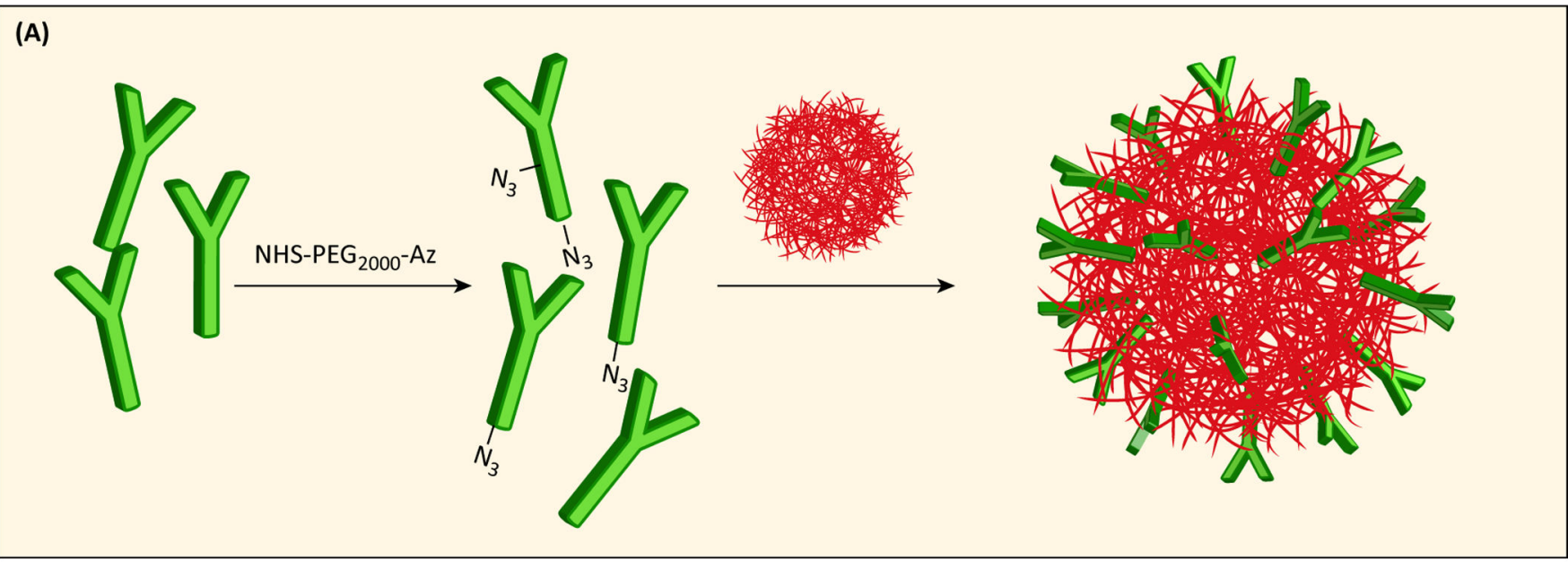

(B)

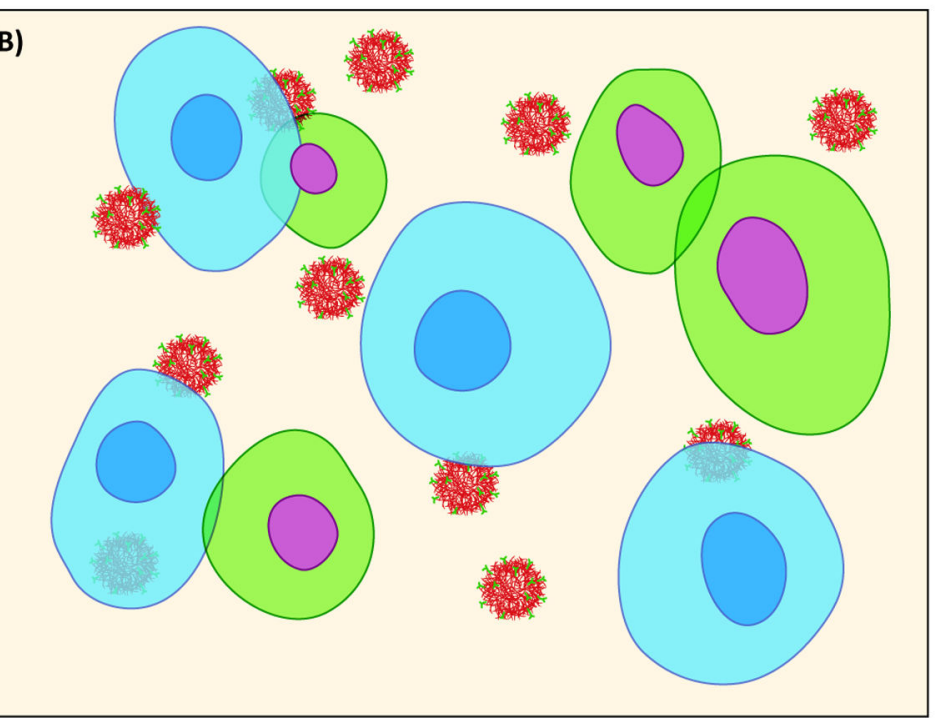

(C)

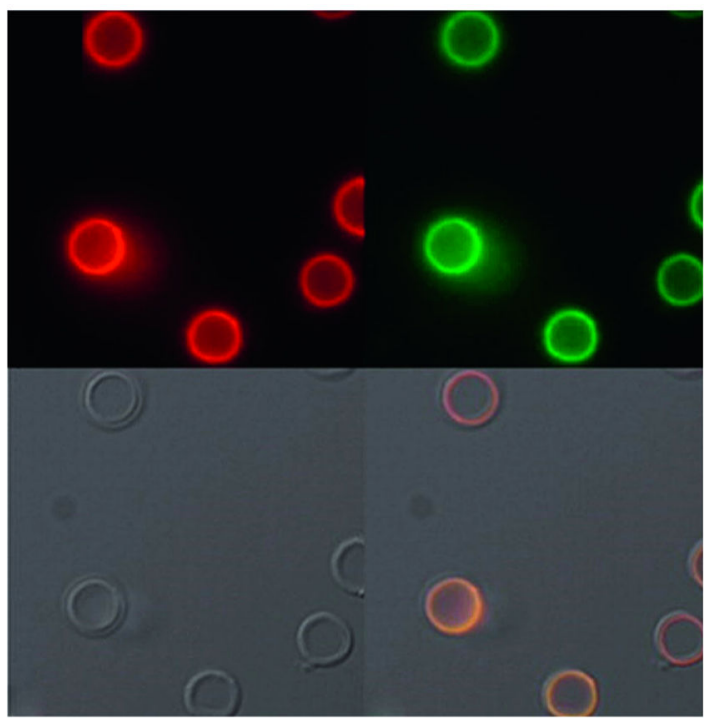

Figure 4.

Antibody functionalization and visualization. (a) Antibodies conjugated to the NP surface through "click" chemistry. (b) Cells that express the complementary antigen are blue and show Ab-facilitated binding of targeted NPs. Cells that do not express the complementary antigen are green with no NP binding. (c) Fluorescence microscopy images of huA33 mAbAzfunctionalized nanocapsules with (i) the antibody labeled with AF647 (red), or (ii) antibody labeled with AF488 (green), (iii) brightfield, and (iv) overlay images. Adapted with permission from [61]. 


\section{Table 1}

Nanomedicines in clinical development

\begin{tabular}{llll}
\hline & $\begin{array}{l}\text { Targeting } \\
\text { ligand }\end{array}$ & $\begin{array}{l}\text { Therapeutic } \\
\text { encapsulated }\end{array}$ & Indication \\
\hline Liposomes & & & $\begin{array}{l}\text { Clinical } \\
\text { status }\end{array}$ \\
\hline ALN-TTR02 (NCT01559077) & Passive & siRNA & Transthyretin amyloidosis \\
\hline CALAA-01 (NCT00689065) & Transferrin & siRNA & Solid tumors \\
\hline CPX-351 (NCT00822094) & Passive & Cytarabine and daunorubicin & Acute myeloid leukemia \\
\hline MBP-426 (NCT00964080) & Transferrin & Oxaliplatin & Pastroesophageal adenocarcinoma \\
\hline SGT53-01 (NCT00470613) & Phase II \\
\hline TKM-PLK1 (NCT01262235) & Antibody fragment & p53 gene & Solid tumors \\
\hline Polymeric NPs & Passive & siRNA & Solid tumors \\
\hline BIND-014 (NCT01300533) & & & Phase I \\
\hline Atu027 (NCT01808638) & Small molecule & Docetaxel & Solid tumors \\
\hline CRLX-101 (NCT01380769)/(NCT00333502)/(NCT02010567) & Passive & Camptothecin & Solid tumors \\
\hline
\end{tabular}

